\title{
Autoeficácia docente: predição da satisfação dos professores
}

\section{Teacher's self-efficacy: predicting the teacher's satisfaction}

\author{
Regina Capelo ${ }^{1}$ \\ Margarida Pocinho ${ }^{1}$
}

\begin{abstract}
RESUMO
Este estudo quantitativo, transversal e preditivo tem como objectivo analisar a influência da autoeficácia na satisfação profissional dos professores portugueses. A amostra é constituída por 327 professores do $1 .^{\circ}$ Ciclo do Ensino Básico (trabalham com alunos dos 6 aos 10 anos) que responderam a um questionário de dados sociodemográficos e escalas validadas para a população portuguesa para as variáveis em estudo. Realizada a análise de regressão linear múltipla, verificou-se que o aumento da "eficácia instrucional" e da "eficácia nos relacionamentos interpessoais" predizem um aumento da satisfação docente na dimensão "relação com a profissão" ( $\mathrm{R}^{2}$ =.27). A uma melhor perceção da "relação interpessoal/institucional" $\left(\mathrm{R}^{2}=\right.$ .20), corresponde uma diminuição da "eficácia instrucional" e um aumento da eficácia nos relacionamentos interpessoais.
\end{abstract}

Palavras-chave: autoeficácia; satisfação profissional; professores.

\begin{abstract}
The aim of this quantitative cross-sectional and predictive study is to analyze the influence of self-efficacy of Portuguese teachers' job satisfaction. A sample of 327 primary teachers from the first grade (working with pupils from 6 to 10 years) answered a sociodemographic questionnaire and Portuguese self-efficacy and job satisfaction validated population scales. A multiple linear regression analysis showed that the increase in "instruction
\end{abstract}

DOI: $10.1590 / 0104-4060.37870$

1 Universidade da Madeira. Centro de Competências de Artes e Humanidades. Funchal, Região Autónoma da Madeira, Portugal. Praça do Município. 9000-082. 
effectiveness" and "effectiveness in interpersonal relationships" predict an increase of teaching satisfaction on the "relationship with the profession" $\left(\mathrm{R}^{2}=.27\right)$. A better perception of "interpersonal/ institutional relationships" $\left(\mathrm{R}^{2}=.20\right)$ corresponds to a decrease in "instructional effectiveness" and an increase on effectiveness in interpersonal relationships.

Keywords: self-efficacy; job satisfaction; teachers.

\section{Introdução}

As transformações aceleradas advindas do impacto causado pela globalização, tecnologia, competitividade e crise, em geral, e a perda de sentimento de coletividade, em particular, têm transformado o trabalho num espaço propiciador de insatisfação profissional, ao qual o grupo docente não está imune. Porém, a literatura tem vindo a realçar o papel harmonizador de diversas variáveis na relação que os professores estabelecem com a profissão, designadamente, a autoeficácia (CAPELO, 2010; CAPRARA; BORGONI; STECA, 2003; PEDRO, 2011; POCINHO; CAPELO, 2009; TSCHANNEN-MORAN; HOY, 2007; VENDITTI, 2005).

O constructo multidimensional, autoeficácia, cunhado por Bandura (1997), que se assenta na crença que o indivíduo tem na sua capacidade de realizar com sucesso as atividades desejadas, tem sido incluído nos estudos sobre o comportamento humano nas organizações. A autoeficácia é tida como um dos mecanismos fundamentais do agenciamento humano (BANDURA, 1997, 2006). Para Bandura (2008), ser agente significa, intencionalmente, influenciar o próprio funcionamento e as circunstâncias da vida. Nesse sentido, as pessoas são auto-organizadas, proactivas, autorreguladas e autorreflexivas, contribuindo para a construção das suas vidas, não sendo, apenas, produtos dessas circunstâncias. Esta perspetiva conduz-nos ao conceito de professor eficaz ligado à ideia de professor motivado, que se realiza pessoalmente através da experiência profissional, e além disso, vive o dia a dia com prazer e interesse pelas tarefas que executa (MADEIRA, 2000).

Nesta linha de pensamento, Schwarzer e Schmitz (2004) ressalvam que um docente com elevados níveis de autoeficácia será um professor muito mais proactivo, na medida em que acredita na existência dos recursos necessários, quer sejam externos quer sejam internos, assumindo uma postura de responsabilidade no que toca ao seu crescimento profissional, que se centra na procura de soluções para os problemas com que se vê confrontado e que define os caminhos 
de ação. Também Bzuneck (2000) acrescenta a estas caraterísticas a definição de objetivos profissionais desafiantes, o dever de compromisso para atingir esses objetivos a que se propôs, e não menos importante, uma atitude construtiva perante os obstáculos e fracassos. Por sua vez, o estudo de Tschannen-Moran e Hoy (2007) apresenta uma associação do conceito de satisfação profissional às experiências pessoais bem-sucedidas, referidas por Bandura (2008), isto é, associa as experiências de sucesso aos sentimentos de satisfação que os professores obtêm no trabalho.

A satisfação no trabalho, na atualidade, tornou-se num dos temas mais relevantes nas organizações pelo interesse na compreensão do fenómeno da satisfação/insatisfação no trabalho devido às implicações significativas que o mesmo produz. Esta problemática também atingiu a organização escolar e os professores. No entanto, apesar de existirem professores insatisfeitos, Pedro (2011) destaca que muitos professores detêm sentimentos satisfatórios na relação que estabelecem com a profissão. Neste sentido, Seco (2002) define satisfação profissional docente como um sentimento que provoca bem-estar para com o ensino, decorrente do nível de adaptação entre a atividade profissional e as características pessoais do professor.

Pedro (2007) remete-nos para a satisfação profissional dos professores como um estado geral positivo e emocionalmente adquirido através das desejadas recompensas auferidas pelo trabalho em ambiente escolar. Por sua vez, salienta que as investigações que se têm efetuado revelam decréscimo do nível de satisfação e reitera que a classe docente não retira satisfação do seu dia a dia profissional, chamando atenção para uma realidade que não é exclusiva de Portugal. Neste domínio, o estudo realizado por Pinto, Lima e Silva (2003) com professores de vários países europeus mostra que os professores portugueses apresentam níveis inferiores de satisfação profissional comparativamente aos professores de outras nacionalidades estudadas. Investigações realizadas em Portugal por Jesus (2005), Pedro e Peixoto (2006) e Seco (2000) vieram confirmar a existência de desconforto e mal-estar profissional nos professores portugueses.

Diversas investigações corroboram a existência de satisfação/insatisfação profissional nos docentes, designadamente, satisfação com o trabalho em si e com a supervisão, no entanto insatisfação com o salário e as oportunidades de promoção (KOUSTELIOUS, 2001), produção de impacto negativo no desempenho docente, insatisfação com as políticas educacionais, administrativas e salários (ADENIKE; OYESOJI, 2010).

Caprara, Barbaranelli, Borgoni e Steca (2003) e Pedro (2011) analisaram a relação entre a autoeficácia e a satisfação profissional dos professores e as suas investigações admitiram que as crenças de autoeficácia são determinantes da satisfação profissional. Na verdade, a satisfação profissional engloba a 
interação de uma multiplicidade de elementos essenciais, sobretudo entre as dimensões: alunos, sociopolítica, relações interpessoais/institucionais, estabilidade e realização pessoal (PEDRO, 2011), em que a perceção do trabalhador sobre a sua relação com o trabalho irá culminar numa componente emocional. Assim, o objetivo deste estudo é analisar a influência preditiva da autoeficácia na satisfação profissional dos professores portugueses.

\section{Método}

\section{Participantes}

Participaram neste estudo 327 professores do $1 .{ }^{\circ}$ Ciclo do Ensino Básico (leccionam com crianças dos 6 aos 10 anos) que trabalham em escolas públicas da Madeira, Portugal - 79\% do género feminino e 21\% são mulheres.

\section{Material}

Com vista a recolher os dados indispensáveis à concessão deste estudo utilizaram-se dois instrumentos: o Questionário de Satisfação no Trabalho para Professores (PEDRO; PEIXOTO, 2006) e a Escala de Autoeficácia dos Professores (PEDRO, 2007) e, ainda um questionário de dados sociodemográficos e profissionais.

Apurou-se a estrutura fatorial com rotação Varimax e consistência interna dos instrumentos (FIELD, 2011). O questionário de satisfação profissional avalia a satisfação profissional geral, os fatores da profissão docente que mais contribuem para a insatisfação/satisfação dos docentes e os scores da satisfação profissional total dos fatores da escala. Extraíram-se quatro fatores aceitáveis (alfa de Cronbach entre .67 e .71). O questionário de autoeficácia, por sua vez, avalia as diferentes dimensões que caracterizam a prática docente. A prova de fiabilidade permitiu a identificação de quatro fatores credíveis (alfa de Cronbach entre .70 e .87$)$.

\section{Procedimento}

O estudo transversal e preditivo realizou-se após autorização governamental e consentimento informado dos participantes. O questionário anónimo garantia o cumprimento dos procedimentos éticos. Distribuíram-se 500 questionários que resultaram na amostra final constituída por 327 questionários válidos, o que revela uma taxa de participação, ou de retorno, de cerca de $65,4 \%$. Recolhidos 
os dados obtidos, foram introduzidos e analisados no SPSS-Statistical Package for Social Science, versão 20.0. Tendo em consideração o objetivo do estudo, a análise recaiu sob a utilização da regressão linear múltipla (RLM) (PESTANA; GAGEIRO, 2005).

\section{Resultados}

De forma a investigar os melhores preditores da satisfação na relação interpessoal/institucional, foi realizada uma regressão linear múltipla. A tabela 1 apresenta um resumo das análises de regressão linear múltipla stepwise para as dimensões da satisfação profissional "relação interpessoal/institucional" (modelo 1) e para a satisfação na relação com a profissão (modelo 2).

TABELA 1 - SÍNTESE DA REGRESSÃO LINEAR MÚLTIPLA PARAAS DIMENSÕES DA SATISFAÇÃO PROFISSIONAL A PARTIR DAS DIMENSÕES DAAUTOEFICÁCIA

\begin{tabular}{clcccc}
\hline Modelo & $\mathrm{R}$ & $\mathrm{R}^{2}$ & $\mathrm{R}^{2}$ ajustado & $\mathrm{F}(4,32)$ & Sig. \\
\hline 1 & .52 & .27 & .27 & 30.32 & .00 \\
2 & .45 & .20 & .20 & 21.30 & .00 \\
\hline
\end{tabular}

Variáveis preditoras: Eficácia na promoção da frequência escolar; Eficácia nos relacionamentos interpessoais; Eficácia na promoção do envolvimento parental; Eficácia instrucional; n = 327 .

A combinação das variáveis para predizer a satisfação na "relação interpessoal/institucional" a partir da autoeficácia revelou-se significativa $\mathrm{F}(4,32)$ $=30.32, \mathrm{p}<.00$. É importante ressaltar que os fatores "eficácia instrucional" e "eficácia nos relacionamentos interpessoais" predizem significativamente a satisfação na relação interpessoal/institucional. $\mathrm{O} \mathrm{R}^{2}$ ajustado foi de .27, isto é, verificou-se que $27 \%$ da satisfação na "relação interpessoal/institucional" pode ser explicado pelas variáveis "eficácia instrucional” em conjunto com a "eficácia na promoção dos relacionamentos interpessoais", como se pode verificar pela equação (tabela 2):

Relação interpessoal/institucional $=1.98+0.57$ X Eficácia nos relacionamentos interpessoais - $0.13 \mathrm{X}$ Eficácia instrucional.

Esta equação significa que a relação interpessoal/institucional aumenta com a eficácia nos relacionamentos interpessoais e tende a diminuir com elevados valores de eficácia instrucional.

Também se apurou que a "eficácia instrucional" e a "eficácia nos relacionamentos interpessoais" emergem como os melhores preditores da satisfação 
na "relação com a profissão", sendo $\mathrm{F}(4,32)=21.30, \mathrm{p}<.00$. O R ${ }^{2}$ ajustado é de .20 , indicando que $20 \%$ da variância da satisfação na dimensão "relação com a profissão" é explicada por este modelo, como se pode verificar pela equação (tabela 2):

Relação com a profissão $=1.15+0.30 \mathrm{X}$ Eficácia instrucional $+0.12 \mathrm{X}$ Eficácia nos relacionamentos interpessoais.

Isso significa que a relação com a profissão aumenta, em primeiro lugar, com a eficácia instrucional e, em segundo lugar, com a eficácia nos relacionamentos interpessoais.

TABELA 2 - COEFICIENTES DE REGRESSÃO LINEAR MÚLTIPLA

\begin{tabular}{|c|c|c|c|c|c|c|}
\hline & $\mathrm{B}$ & $\mathrm{DP}$ & $\beta$ & $t$ & $p$ & $\mathrm{~F}(4.32)$ \\
\hline $\begin{array}{l}\text { Constante1: relação } \\
\text { interpessoal/ institucional }\end{array}$ & 1.98 & .24 & & 8.11 & .00 & \multirow{3}{*}{$30.32 * * *$} \\
\hline Eficácia instrucional & -.156 & .08 & -.13 & -1.97 & .05 & \\
\hline $\begin{array}{l}\text { Eficácia nos relacionamentos } \\
\text { interpessoais }\end{array}$ & .62 & .06 & .57 & 10.09 & .00 & \\
\hline $\begin{array}{l}\text { Constante 2: relação com a } \\
\text { profissão }\end{array}$ & 1.15 & .26 & & 4.43 & .00 & \multirow{3}{*}{$21.30^{* * *}$} \\
\hline Eficácia instrucional & .35 & .08 & .30 & 4.18 & .00 & \\
\hline $\begin{array}{l}\text { Eficácia nos relacionamentos } \\
\text { interpessoais }\end{array}$ & .13 & .06 & .12 & 1.99 & .04 & \\
\hline
\end{tabular}

$* * * \mathrm{p}<.001$

1 - variável dependente: relação interpessoal/institucional; 2 - variável dependente: relação com a profissão.

\section{Discussão}

Nos últimos tempos têm ocorrido sucessivas mudanças societárias, às quais o sistema educativo não ficou alheio, que acarretaram um acréscimo nas atribuições docentes, e com consequências para a satisfação profissional. Neste seguimento, Galti (2013) assinala questões sobre o papel do professor do ensino básico perante os desafios socioculturais que lhe são colocados, o que leva a uma discussão sobre a formação inicial deste grupo profissional. Porém, diversos investigadores verificaram que, apesar da inquestionável complexidade da profissão docente, existem profissionais satisfeitos com a profissão (PEDRO, 2007, 2011; SECO, 2002). Ainda com base em investigações, Pedro (2011) explicita algumas variáveis psicológicas fundamentais que podem contribuir para 
a satisfação dos professores, entre as quais se destacam as diferentes dimensões inerentes às crenças de autoeficácia docente. Também no presente estudo, através do cálculo do modelo da regressão linear múltipla, foi possível observar que a eficácia instrucional e a eficácia nos relacionamentos interpessoais constituem preditores da satisfação na relação com a profissão. Já no que diz respeito à relação interpessoal/institucional, a eficácia nos relacionamentos interpessoais tende a aumentar a perceção positiva desta relação, mas a eficácia instrucional tende a diminuir essa mesma perceção. Assim, a eficácia instrucional aumenta a percepção na relação com a profissão, mas diminui a percepção na relação interpessoal.

Estes resultados vêm de encontro ao exposto por Caprara, Barbaranelli, Steca e Maloni (2006) quando defendem que as crenças de autoeficácia dos professores são preditores da (in)satisfação profissional docente. Os mesmos autores salientam que a influência das crenças de autoeficácia decorre simultaneamente, de forma direta, da satisfação das necessidades pessoais de competência, e indiretamente de desempenhos profissionais nos professores adequados e com êxito, conducentes a favoráveis recompensas e a níveis de autoestima elevados.

Neste âmbito, os trabalhos desenvolvidos por Sharma e Jyoti (2006), Pedro e Peixoto (2006) realçam a importância das boas relações estabelecidas com os colegas como um dos fatores justificativos da satisfação profissional. Posteriormente, o estudo de Pedro (2011) colocou em destaque o efeito preditivo da "eficácia nos relacionamentos interpessoais" como preditor importante, explicando $40 \%$ da variância da satisfação profissional. Por sua vez, o dito estudo apresenta a "eficácia instrucional" responsável por 5\% da variabilidade da satisfação profissional. Segundo Pedro (2007), a autoeficácia docente quando adicionada a níveis elevados de satisfação profissional contribui para o sustento do envolvimento, investimento, motivação e dedicação por parte do professor face à sua profissão, bem como levará o mesmo a delinear e a perseguir os objetivos e metas definidos por ele próprio no campo profissional. Sendo assim, apoiando-nos em Bandura (2006; 2008), Fernández-Ballesteros, Díez-Nicolás, Caprara, Barbaranelli e Bandura (2004) e Pedro (2007, 2011), deduzimos que as crenças de autoeficácia partilhadas pelos docentes poderão ser um contributo para a promoção do ambiente profissional direcionado para a obtenção do sucesso educativo dos alunos e do bem-estar psicológico dos docentes.

Um dos aspetos a destacar é a exiguidade de estudos que abordem o grupo docente participante nesta investigação e que nos permitam comparar ou contrastar os resultados obtidos. No entanto, acreditamos que os resultados apresentados trazem algo de inovador à investigação sobre este grupo profissional (professores do $1 .^{\circ}$ Ciclo do Ensino Básico) e poderão contribuir para uma melhor perceção deste grupo docente ou até servir como ponto de partida para futuros estudos 
que envolvam as variáveis aqui discutidas. Isto porque, tendo em conta o efeito multiplicador das crenças na autoeficácia partilhada, desejamos que as nossas escolas incluam nos seus quadros de pessoal professores que possuam crenças de autoeficácia elevada, satisfeitos na sua relação com a profissão, e sobretudo, mais aptos para lidarem com a multiplicidade dos papéis que têm de assumir e com a complexidade das ocorrências do quotidiano escolar.

\section{REFERÊNCIAS}

ADENIKE, A.; OYESOJI, A. The relationship among predictors of child, family, school, society and the government and academic achievement of senior secondary school students in Ibadan, Nigeria. Procedia-Social and Behavioral Sciences, Netherlands, v. 5, p. 842-849, 2010.

BANDURA, A. Self-efficacy: The exercise of control. New York: Freeman, 1997.

BANDURA, A. The Growing Primacy of perceived efficacy in human self-development, adaptation and change. In: SALANOVA, M.; GRAU, R.; MARTÍNEZ, I. M.; CIFRE, E.; LLORENS, S.; GARCÍA-RENEDO, M. (Eds.). Nuevos Horizontes en la Investigación sobre la autoeficacia. Castellón: Colección Psique, 2004. p. 34-51.

BANDURA, A. Toward a psychology of human agency. Perspectives on Psychological Science, London, v. 1, n. 2, p. 164-180, 2006.

BANDURA, A. A evolução da teoria social cognitiva. In: BANDURA, A.; AZZI, G.; POLYDORO, S. (Org.). Teoria social cognitiva: conceitos básicos. Porto Alegre: Artmed, 2008. p. 15-41.

BZUNECK, J. A. As crenças de auto-eficácia dos professores. In: SISTO, F. F.; OLIVEIRA, G. C.; FINI, L. T. (Orgs.). Leituras de Psicologia para a formação de professores. Petrópolis: Vozes, 2000. p. 115-134.

CAPELO, R. Estrés, coping y autoeficacia en profesores de Madeira. 142f. Tese (Doutoramento em Ciências do Trabalho) - Faculdade de Ciencias del Trabajo, Universidad de Cádiz, Cádiz, 2010.

CAPRARA, G. V.; BARBARANELLI, C.; BORGOGNI, L.; STECA, P. Efficacy beliefs as determinants of teachers' job satisfaction. Journal of Educational Psychology, Washington, v. 95, n. 4, p. 831-832, 2003.

CAPRARA, G. V.; BARBARANELLI, C.; STECA, P.; MALONI, P. S. Teachers' selfefficacy beliefs as determinants of teachers' job satisfaction and students' academic 
achievement: A study at the school level. Journal of School Psychology, Netherlands, v. 44, p. 473-490, 2006.

FERNÁNDEZ-BALLESTEROS, R.; DÍEZ-NICOLÁS, J.; CAPRARA, G. V.; BARBARANELLI, C.; BANDURA, A. Determinantes y relaciones estructurales desde la eficacia colectiva. In: SALANOVA, M.; GRAU, R.; MARTINEZ, I. M.; CIFRE, E.; LORENS, S.; GARCÍA-RENEDO, M. (Eds.). Nuevos Horizontes en la Investigación sobre la autoeficácia. Castellón: Colección Psique, 2004. p. 68-80.

FIELD, A. Discovering statistics using SPSS. 3. ed. London: SAGE, 2011.

GALTI, B. Educação, escolar e formação de professors: políticas e impasses. Educar em Revista, Curitiba, n. 50, p. 51-67, 2013.

GARCÍA, M.; LLORENS, S.; CIFRE, E.; SALANOVA, M. Antecedentes afectivos de la autoeficacia docente: un modelo de relaciones estructurales. Revista de Educação, Lisboa, v. 339, p. 387-400, 2006.

JESUS, S. N. Bem-estar docente. Perspectivas para superar o mal-estar dos professores. In: SILVA, A. L.; PINTO, A. M. (Eds.). Stress e bem-estar. Lisboa: Climepsi Editores, 2005. p. 167-184.

KOUSTELIOUS, A. Organizational facts as predictors of teachers' burnout. Psychological Reports, Missoula, v. 88, p. 627-634, 2001.

LOUREIRO, C. A docência como profissão. Porto: Edições ASA, 2001.

MADEIRA, H. M. A importância da motivação na instituição escolar. O professor, v. 69, p. 64-68, 2000.

PEDRO, N. A auto-eficácia e a satisfação profissional dos professores. $177 \mathrm{f}$. Dissertação (Mestrado em Psicologia da Educação) - Instituto Superior de Psicologia Aplicada, Lisboa, 2007.

PEDRO, N. Auto-eficácia e satisfação profissional dos professores: colocando os constructos em relação num grupo de professores portugueses. Revista de Educação, Lisboa,v. XVIII, n. 1, p. 23-47, 2011.

PEDRO, N.; PEIXOTO, F. A satisfação profissional e auto-estima dos professores. Análise Psicológica, Lisboa, v. 24, n. 2, p. 247-262, 2006.

PESTANA, M.; GAGEIRO, J. Análise de dados para Ciências Sociais: A complementaridade do SPSS. Lisboa: Edições Sílabo, 2005.

PINTO, A. M.; LIMA, M. L.; SILVA, A. L. Stress profissional em professores portugueses: Incidência, preditores e reacção ao burnout. Psychologica, Coimbra, v. 33, p. 181-194, 2003.

POCINHO, M.; CAPELO, R. Vulnerabilidade ao stresse, estratégias de coping e auto-eficácia em professores portugueses. Educação e Pesquisa, São Paulo, v. 35, n. 2, p. 351-367, 2009. 
SCHWARZER, R.; HALLUM, S. Perceived teacher self-efficacy as a predictor of job stress and burnout. Mediation Analyses. Applied Psychology: An International Review, Malden, v. 57, p. 152-171, 2008.

SCHWARZER, R.; SCHMITZ, G. S. Perceived self-efficacy as a resource factor in teachers. In: SALANOVA, M.; GRAU, R.; MARTÍNEZ, I. M.; CIFRE, E.; LLORENS, S.; GÁRCIA-RENEDO, M. (Eds.). Nuevos horizontes en la investigación sobre la autoeficacia. Castelló de la Plana: Publicaciones de la Universitat Jaume I, 2004. p. 229-236.

SECO, G. B. A satisfação na atividade docente. Coimbra: Universidade de Coimbra, 2000.

SECO, G. B. A satisfação dos professores: Teorias, modelos e evidencias. Porto: Edições ASA, 2002.

SHARMA R. D.; JYOTI, J. Job satisfaction among school teachers. IIMB Management Review, Bangalore, p. 349-363, 2006.

TSCHANNEN-MORAN, M.; WOOLFOLK HOY, A. The differential antecedents of self-efficacy beliefs of novice and experienced teachers. Teaching and Teacher Education, Netherlands, v. 23, p. 944-956, 2007.

VENDITTI, R. J. Análise da autoeficácia docente de professores de educação física. 167 f. Dissertação (Mestrado em educação Física) - Faculdade de Educação Física, Universidade Estadual de Campinas, São Paulo, 2005.

Texto recebido em 21 de setembro de 2014. Texto aprovado em 25 de setembro de 2014. 